\title{
Impfung - allein die Dosis macht's?
}

Vaccination - It's All About the Right Dosis?

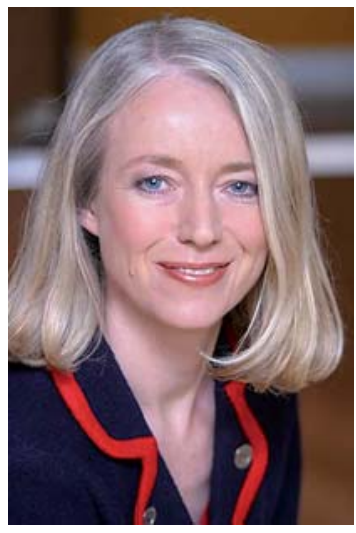

Prof. Dr. Christiane Bayerl
Seit Edward Jenner (1749-1823) und der Pockenimpfung und der prophylaktischen Impfung gegen HPV-assoziierte Neoplasien, verknüpft mit dem Namen zur Hausen und dem Nobelpreis Medizin 2008, sind drei Jahrhunderte vergangen. In diesem Heft finden Sie eine Übersichtsarbeit zur HPV-Impfung. Die Dosis ist bei Vakzinen nicht das einzig Entscheidende, es sind komplexere Entwicklungen, die hinter derartig bahnbrechenden Neuerungen stehen.

Ungeklärt ist die Dosisfrage bei der therapeutischen Hyposensibilisierung - muss es wirklich eine kumulative Gesamtdosis sein, die den Effekt einer Hyposensibilisierung auf z.B. Pollen ausmacht? Diese Frage ist noch nicht abschließend geklärt.

Vernachlässigt wird bei den üblichen präventiven Routineimpfungen wie z.B. bei Tetanus die individuelle Reaktion der Antikörperbildung bei einer Komplikation. Kurz vor der dritten Impfung oder der Auffrischimpfung nach 10 Jahren erinnert sich der Patient an die Unverträglichkeitsreaktion bei der letzten Impfung. Wünschenswert wäre aber eine zeitnahe Abklärung. Die Palette der möglichen Komplikationen umfasst Impfreaktionen und Impfkomplikationen. Impfreaktionen sind die erwarteten Komplikationen wie z.B. Schmerzen an der Injektionsstelle, Müdigkeit, Abgeschlagenheit, Kopfschmerzen, kurz eine Grippe-ähnliche Symptomatik. Ausgeprägte Lokalreaktionen bis hin zur Arthus-Reaktion, Abszessbildungen und anaphylaktische Reaktionen werden beobachtet, sind aber insgesamt seltene Ereignisse - so die Statistik. Es besteht Meldepflicht, aber die Erfassung hängt eben an unserer Motivation zu melden. 0,2\% der im Paul-Ehrlich-Institut gemeldeten Fälle fallen unter die Kategorie Impfkomplikationen. Einem Allergologen scheinen diese Daten nicht ganz glaubhaft, finden wir doch gerade die Spitze des Eisberges, nämlich Allergien auf Quecksilberverbindungen (Thiomersal), Formaldehyd, Antibiotika, Hühnereiweiß oder Gelatine, als Ursache für anaphylaktische oder verzögerte Impfreaktionen. Von verzögerten Reaktionen abzugrenzen sind die Arthus-Reaktio- nen oder Serum-Krankheitsreaktionen, 4-10 Tage nach der Impfung als lokale Typ-III-Hypersensitivitätsreaktion bei Immunkomplexbildung mit Ablagerung der Komplexe in der Gefäßwand, Pleura, Perikard, Synovia, Glomeruli bis hin zur Gangrän an der Injektonsstelle gerade bei zu früh erneut geimpften Patienten. Daher wurde empfohlen auf keinen Fall vor 10 Jahren eine Auffrischung der Tetanusimpfung durchzuführen (siehe STIKO-Empfehlungen). Berichte über systemische Reaktionen (Urtikaria, Angioödeme, Exanthem, respiratorische Symptome) eine Stunde nach Enzephalitisimpfungen beruhten auf erhöhtem spezifischem IgE und in einigen Fällen auch IgG gegen Gelatine [1]. Nach einer FDA-Empfehlung werden Hypersensitivitätsreaktionen in 6 Kategorien eingeteilt [2]: 1. Reaktionen gegen Komponenten des infektiösen Agens, 2. Reaktionen auf Adjuvantien wie Aluminiumhydroxid, 3. Reaktionen auf Stabilisatoren wie Gelatine, 4. Reaktionen gegen Konservierungsstoffe wie Thiomersal, 5. Reaktionen auf Antibiotika wie Neomycin, 6. Reaktionen auf ein biologisches Kulturmedium wie Hühnereizellen. Nicht zu impfen ist selbstverständlich keine adäquate Antwort. Üblicherweise kann eine Impfung selbst bei Ei-Allergikern sicher durchgeführt werden, wenn die Dosis gesplittet wird und vor allem Notfallbereitschaft und Überwachung ärztlicherseits gewährleistet ist [3].

Ihre

Christiane Bayerl

\section{Literatur}

1 Sakaguchi M, Miyazawa H, Inouye S. Specific IgE und IgG to gelatin in children with systemic cutaneous reactions to Japanese encephalitis vaccines. Allergy 2001; 56: 539-539

2 Eseverri J, Ranea S, Marin A. Adverse reactions to vaccines. Allergol Immunopathol 2003; 31: 125-138

3 Schuler JE, King WJ, Dayneka NL et al. Administration of the adjuvanted pH1N1 vaccine in egg-allergic children at high risk for influenza A/H1N1 disease. Can J Public Health 2011; 102: 196-199
DOI http://dx.doi.org/

Akt Dermatol 2011; 37; 235

(c) Georg Thieme Verlag KG

Stuttgart · New York

ISSN 0340-2541

Korrespondenzadresse

Prof. Dr. med. Christiane BayerI

Klinik für Dermatologie

und Allergologie

HSK, Wilhelm-Fresenius-Klinik

Städtisches Klinikum Wiesbaden

Aukammallee 39

65191 Wiesbaden

christiane.bayerl@

hsk-wiesbaden.de 